\title{
The analysis of dispersed generation influence on power system automatics settings and function algorithms
}

\author{
Pavel Ilyushin ${ }^{1 *}$ \\ ${ }^{1}$ Petersburg power engineering institute of professional development, Aviation str. 23, Saint-Petersburg, Russia
}

\begin{abstract}
Dispersed generation (DG) objects are usually connected to distribution grid. This-type of grid supposes to have power system automatics (PSA) that comprise emergency control system (ECS). ECS serves for normal power supply scheme recovery after fault elimination, fault development prevention and system parameters maintenance in acceptable limits within fault and post-fault conditions. Generating power connection to the grid causes significant power flow and current distribution change. This might become a reason of ECS automatics misoperation. This paper presents the results of DG objects influence analysis on PSA settings and function algorithms. Implemented analysis has allowed to define post-fault conditions envelopes that have to be analysed within DG objects grid connection design and provide the recommendations for PSA settings and function algorithms change.
\end{abstract}

\section{Introduction}

At present, the tendency of distributed generation (DG) objects commissioning increase is resuming in Russia. They are usually built by the owners of industrial enterprises in the oil and gas, mining, metallurgical, pulp and paper and chemical industries. They are connected to distribution electric grids or to internal power supply networks of these enterprises.

At the same time, power system automatics are widely used in these grids: automatic switching of backup power supply (ABS), automatic frequency discharge (AFD) and automatic control of voltage reduction (ACVR). These devices are used to restore the normal circuit of power supply, prevent the development of accidents, as well as normalize the parameters of the electrical mode in emergency and post-emergency circuit-mode situations. They are necessary to ensure the normal operation of the most part of consumers' electric receivers.

The connection of small and medium power generating units (GUs) of DG objects inevitably leads to a change in the circuit-mode situations in the grid, significantly affecting, in some cases, the automation devices of the power systems in operation. This applies both to the correctness of their operation, in accordance with the specified algorithms, and to the correct tripping, in accordance with the setpoints. It is important to avoid failures in the operation, as well as excessive and false trips of power automation devices.

Therefore, when developing the project of technological connection (TC) of the DG facility, it is necessary to conduct an analysis of the correctness of the technical solutions for the operation algorithms and the adjustment of the previously installed units of power systems automation in the adjacent grid. For this, it is necessary to make calculations of electrical modes, including calculations of stable modes and electromechanical transient processes. In addition, it is necessary to check the consistency of the operation algorithms and the settings of the automation devices of the power systems in operation with the algorithms of operation and the settings of the new automation devices that must be put into operation at the $\mathrm{TC}$ of the DG object.

\section{Characteristics of ABS operation in the grids with DG}

Automatic backup power and equipment switching devices (ABS) are used to restore power of power receiving installations. This is done by automatically connecting a backup power source when the consumer's electrical installations are de-energized and switching on the backup equipment when the working equipment is disconnected with a violation of the normal process. ABS devices can be installed on transformers, power lines, sectional and bus breaker, electric motors, etc. [1].

If the circuit-mode situation and the disturbance are such that the ABS can not trip due to the operational input disconnection (the input does not turn off) and therefore trips due to the voltage drop, it is clear that the presence of a GU on the redundant part of the network increases the residual voltage. This will interfere with the normal operation of the ABS device. Let us consider the case when the GUs are equipped with a system of independent excitation, which provides for the maximum forcing value independent of the voltage at the GU terminals, and are not de-energized due to the voltage drop at short-circuit.

Corresponding author: ilyushin.pv@mail.ru 
The main parameter determining the critical time for the dynamic stability of the GU is the magnitude of the mechanical constant of inertia of the GU, $T_{J}$, s. $T_{J}$ values close to $1 \mathrm{~s}$ are typical for gas piston units (GPU) and three-shaft gas turbine units (GTU), about 3 seconds for high-power GPUs and twin-shaft gas turbines, 6-8 seconds for small and medium-sized steam turbine generators, 10-12 c - for single-shaft gas turbines. The value of $T_{J}$ in the calculations is assumed to be $2 \mathrm{~s}$, which is typical for the GU of the DG objects.

Calculations show that in many cases, with different combinations of load and generation $(0<d<100 \%, 0<p$ $<100 \%$ ), the operation of electric receivers is violated. The areas of possible post-accidental modes (PAM) are shown in Fig. 1.

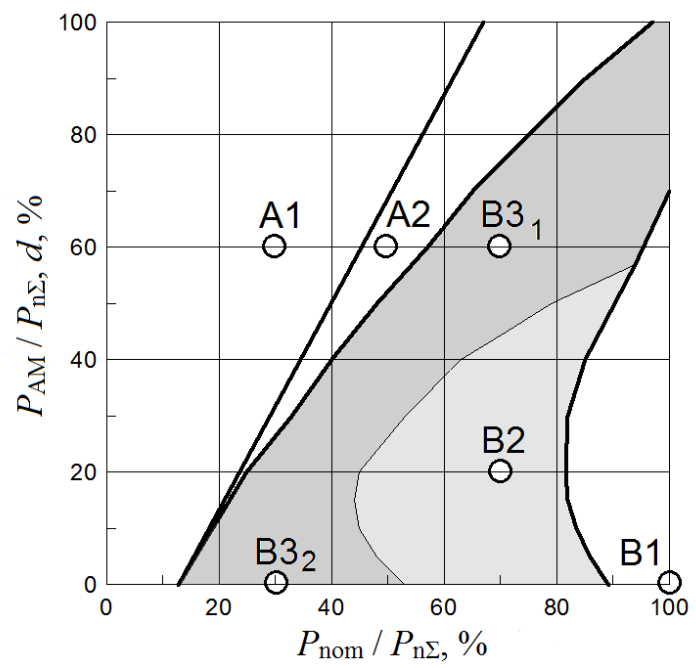

Fig. 1. Post-emergency modes when ABS is triggered by a voltage drop.

In areas where $\mathrm{ABS}$ devices operate, the following are possible:

- Normal trip (the initial parameters of the mode correspond to the area A1),

- Increase in the response time of the ABS due to the fact that the GUs increase the voltage and the voltage starting point (VSP) does not trip until the braking of the asynchronous motors (AM) causes a sufficient voltage drop (area A2).

The failure of the ABS device can result in:

- The modes where the initial parameters correspond to the area B1, which can be considered as short-time permissible voltage (above the critical values) and frequency (conditionally accepted $\mathrm{f}>45 \mathrm{~Hz}$ ). These modes can appear due to a decrease in the power consumed by the AM and synchronous motors (SM) with a decrease in frequency, as well as by static electric receivers with a decrease in voltage. They are limited by the permissible duration of the forced GU excitation (20$30 \mathrm{~s}$ ) and the triggering of the AFD devices,

- The modes corresponding to area B2, where the frequency decreases, but the voltage, due to the forced excitation of the GU, remains above the critical one,

- The occurrence of a voltage collapse - area B3.
In the $\mathbf{B 3}$ area, when there is a high probability of a voltage collapse, triggering of the ABS is necessary in this case, but:

- There may be a failure in the operation of the VSP, since in the case of a voltage collapse, it decreases in different ways, and its effective values may contain variable components,

- May be unacceptable, since the closure of the link between substations (SS), where a voltage collapse has just passed and normally operating SS, can cause the spread of a voltage collapse to these substations and lead to the disconnection of an additional number of consumers [2].

The transient process in area $\mathbf{B 3}$ (point $\mathbf{B 3}_{1}$ ), where $d$ $=60 \%, p=70 \%$, is characterized by the fact that the voltage after the elimination of short-circuit faults does not recover above $48 \%$ of $U_{\text {nom. }}$, which leads to the disconnection of a significant number of electric receivers, while the frequency in the power district rises to $52 \mathrm{~Hz}$.

The transition process in the $\mathbf{B 3}$ area (point $\mathbf{B 3}_{\mathbf{2}}$ ), where $d=0, p=30 \%$, is characterized by the fact that the voltage after the elimination of short-circuit is restored to $70 \%$ of $\mathrm{U}_{\text {nom, }}$ which leads to a smooth decrease in frequency, considering the significant shortage of active power, of a power district from 50 to $24 \mathrm{~Hz}$ for $4 \mathrm{~s}$ from the beginning of the fault (without taking into account the operation of the AFD).

Remote and incomplete phase short-circuits result in the failure of the ABS to such PAM, which are more favorable, since at the time of such a short-circuit the motors are braked less than in the case of a near threephase short-circuit.

Taking into account the above said, the operation of ABS devices due to the voltage decrease, adjusted as delayed from the trip of relay protection devices (RP), ABS devices located closer to the power supply and automatic reclosing devices does not meet the requirements for the speed required in the presence of DG facilities in the power center.

The increase in the speed of the ABS is possible due to the use of launching units that monitor the parameters of the previous mode, detecting the trip of the RP devices and the current state of the switching devices in the grid, including GU breakers. At the stage of the technological connection project development, on the DG object it is required to verify the correct operation of all ABS devices in the grid of external and internal power supply and, if necessary, provide for the implementation of the necessary organizational and technical measures.

\section{Features of AFD operation in grids with DG}

Automatic frequency discharge (AFD) is designed to prevent unacceptable frequency reduction according to the standards of stable operation of generating equipment and power-receiving units and its subsequent recovery [3]. The operation of the ABS devices and the automatic reclosure must be consistent with the 
operation of the AFD devices in such a way that their action does not restore the power to the consumer load, disconnected by the AFD devices, from the same or other electrically connected power supplies. The tripping of AFD devices in transient modes characterized by frequency reduction, not associated with an emergency shortage of active power or with an interruption in power supply should be avoided [4].

Obviously, the consequences of a power district outage from the power system, in which the GU facilities of the DG can not cover the entire load, are determined by a combination of two factors: a shortage of active power, which leads to frequency decrease, and a shortage of reactive power, which leads to voltage decrease and can trigger a voltage collapse. The latter, if it covers the entire energy district, will make it impossible for all electric receivers to work.

Prior to the commissioning of the DG objects the consumers' frequent cases were:

- Violation of power supply to the power district quickly led to deep voltage failures and the normal operation of the ABS,

- The disconnection of a small part of the generation in the power system or its island part was accompanied by a frequency decrease while maintaining the voltage at an acceptable level with the AFD effect [5].

In the intermediate case, when the energy district is not large and contains the DG object, during the separation from the power system, there can be an arbitrarily large shortage of active and reactive powers. Currently, there are likely qualitative differences between the processes under consideration from the same processes to the construction of DG objects with modern GUs having small $T_{\mathrm{J}}$ values, as indicated earlier. Differences are due to two circumstances:

- The rate of frequency reduction increases, which may require more speed of the AFD devices,

- If the power shortage is significant, then the probability of voltage collapse occurrence substantially increases.

In the latter case, if the voltage fails deep and fast, a very significant load drop is obtained and the balance of active power can be restored, which will lead to normalization of the frequency, while the AFD will not work. In addition, the voltage collapse can pass so quickly that it is impossible to prevent it with ACVR devices.

Consider a small power district with an industrial load, which, in addition to the static load, includes the $\mathrm{AM}$ and the DG object. The power district is connected to the power system by a transmission line, while the voltage on the buses of the power system does not depend on the processes in this power district. In the initial mode, the share of AM in the total load varies and is $d=P_{\mathrm{AM}} / P_{\mathrm{n} \Sigma}, \%$. The value of the generation varies and is set relative to the value of the total load $\left(P_{\text {nom }} /\right.$ $\left.P_{\mathrm{n} \Sigma}, \%\right) ; T_{\mathrm{J}}=2 \mathrm{~s}$; loading of $\mathrm{GU}$ in the initial mode is nominal, short-term available power is $110 \%$; the GU excitation is independent, the GU parameters are the same as for the $2 \mathrm{MW}$ GPU. In the initial mode, the reactive power is set equal to $0.5 P_{\text {nom }}$, the initial voltage on the load buses is $10.2 \mathrm{kV}$.

If the connection between the power district and the power system is broken, the calculations show that the primary factors determining the course of the process with loss of external power are the values of the initial active power shortage $D_{\mathrm{P}}=1-P_{\mathrm{n}} / P_{\mathrm{n} \Sigma}(\%)$ and the share of AM $d(\%)$. In these coordinates, the process can in principle be represented by four areas $\mathbf{A}, \mathbf{B}, \mathbf{C}, \mathbf{D}$, as shown in Fig. 2.

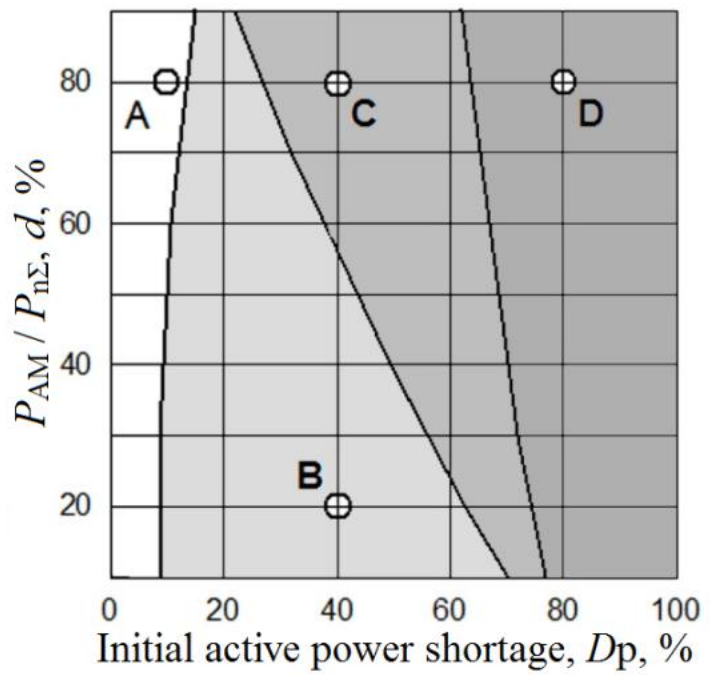

Fig. 2. Features of the processes in the islanding of the energy district with the DG object.

In area $\mathbf{A}$, successful processes: in stable modes $U>$ $0.8 U_{\text {nom }}, \mathrm{f}>49 \mathrm{~Hz}$, which is due to the presence of short-term reserves of the GU (overload of the drive gas piston engines and the excitation forcing) and the regulating effects of the load, linearly dependent on $\mathrm{d}$ (for $d=0$, the control effects $K_{\mathrm{U}} \approx 2, K_{\mathrm{f}}=0$; if $d=100 \%$, then $K_{\mathrm{U}} \approx 0, K_{\mathrm{f}} \approx 1.8$ ).

Area $\mathbf{B}$ corresponds to the conditions when the GU of the DG object keeps the voltage above the critical value, the AM continues to operate, the available power of the GU is insufficient and the frequency decreases; the share of AM is not large and they can not provoke a voltage collapse. This is the circuit-mode situation for which AFD devices are designed.

Area $\mathbf{C}$ is the same as $\mathbf{B}$, but the share of $\mathrm{AM}$ is larger, so the increase in their reactive power consumption (as the frequency decreases) leads to a significant decrease in voltage.

Area D corresponds to a very large initial power shortage and quickly causes the occurrence of a voltage collapse, the AM stop. When the voltage is about $10 \%$ of the rated voltage, the total load is small and the GU speed controllers provide for a frequency increase in the power district to the nominal value.

The described processes refer to cases when at the beginning of the process there is no significant voltage drop. If the emergency mode starts with a short-circuit, the rapid braking of the motors increases the total reactive load and reduces the voltage faster, in comparison with the case of absence of short-circuit. The 
consequence is a decrease in the active load and a lower frequency decrease in the power district.

The same effect - the expansion of the $\mathbf{D}$ area - is manifested if the GUs on the DG object have not an independent excitation system, as in the previous cases, but a self-excitation system in which the maximum possible excitation degree decreases in proportion to the reduced voltage at the GU terminals. At the initial moment, the short-circuit voltage decreases after a few seconds to almost zero, and a full load drop occurs, which leads to a frequency increase. Thus, in the $\mathbf{D}$ area, the size of which depends on the parameters of the power district and the initial disturbance, the AFD devices can not serve as the main means of normalizing the mode in the power district [6].

Taking into account the above said, at the stage of technological connection project development of the DG object it is required to conduct an analysis of the AFD efficiency in the power district when the communication with the power system is broken and, if necessary, provide for the installation of additional emergency control system (ECS) for normalizing the parameters of the mode and keeping the largest part of the consumer electric receivers operational.

\section{Features of ACVR operation in the grids with DG}

Automation of voltage reduction limitation (ACVR) is designed to prevent the violation of load stability and the occurrence of a voltage collapse in post-emergency modes of the power system. It should effect the excitation forcing of synchronous machines, compensation devices forcing, reactors shutdown and as an exception, and in case of insufficient network activities and the existence of a justification - the consumers switch off [1].

The initial conditions for selecting the ACVR devices setting parameters that act to disconnect consumers' electric receivers or their groups are as follows:

- The criterion by which the control action (CA) to disconnect the electric receivers is formed is the voltage after the RP devices of the grid eliminate the emergency mode by disabling the damaged element (controlled voltage),

- ACVR trip should occur if the reference voltage is below the critical voltage ${ }^{a}$ for a given load (with an appropriate redundancy),

- ACVR trip should not occur with direct motor starts and other disturbances in normal production processes at the customer site,

- There should be no unnecessary disconnections of consumers' electrical receivers.

It follows from item 1 that the realization of the recovery should be made after the occurrence of a voltage failure with a time delay, the value of which determines the slowest RP device, on which the recovery of the monitored voltage depends. It is obvious that the

\footnotetext{
${ }^{a}$ The critical voltage is the minimum voltage at which the electrical receivers can continue to perform their functions.
}

possibility of electric motors self-start and, correspondingly, the necessary amounts of their shutdown depend on many factors, and not only on the value of the controlled voltage at the time when the ACVR device trips [7].

The need to increase the efficiency of ACVR devices is dictated by the load properties, the value of the damage from violations of the production process and statistical data on the frequency of occurrence of violations in electrical grids. The use of complex and high-precision technological lines of foreign manufacturers in domestic industrial enterprises and the presentation of high quality requirements for products to enter the world markets necessitates the provision of quality in power supply systems in accordance with the requirements of GOST 32144-2013 [8]. And this, in turn, entails the need to improve the principles of emergency control.

The ACVR device, which enables the recovery only by load shedding (LS) with a time delay of 6-22 s, may not be very effective or completely ineffective if a group of electric motors of considerable power is decelerated. In this case, the longer the voltage is reduced, the higher the total starting current of the motors, the lower the voltage, and the greater the probability will be that the process will end with a voltage collapse. Obviously, the earlier the shutdown of a part of the electric motors is performed, the smaller the amount of load disconnection is required.

Consider an example of calculations performed for a real industrial enterprise with large number of electric motors in the load. The total power consumption of the unit is about $10 \mathrm{MW}$, about $90 \%$ of it is by AM. The load is supplied from a powerful TPP through a $6 \mathrm{kV}$ distribution grid (external to the unit under consideration); the total equivalent resistance of the external grid, reduced to a voltage of $6 \mathrm{kV}, \mathrm{Z}_{\mathrm{eq}} \approx 0.6$ Ohm. The load unit contains a DG object with a $2 \mathrm{MW}$ GPU. In the calculated processes, due to a three-phase short-circuit with a duration of $0.2 \mathrm{~s}$, the connection between the power system and the specified unit is loosened (the resistance $\mathrm{Z}_{\mathrm{eq}}$ is multiplied by 1.5).

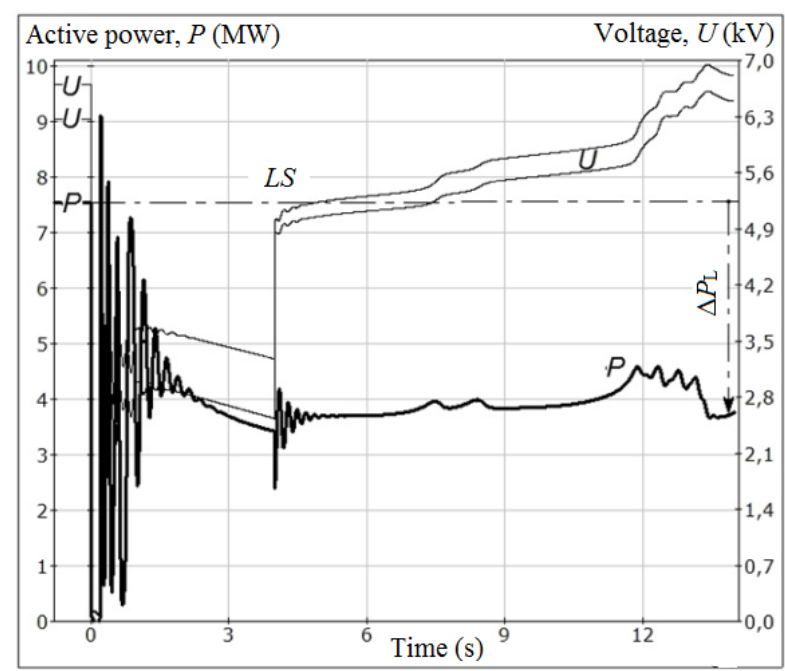

a) 


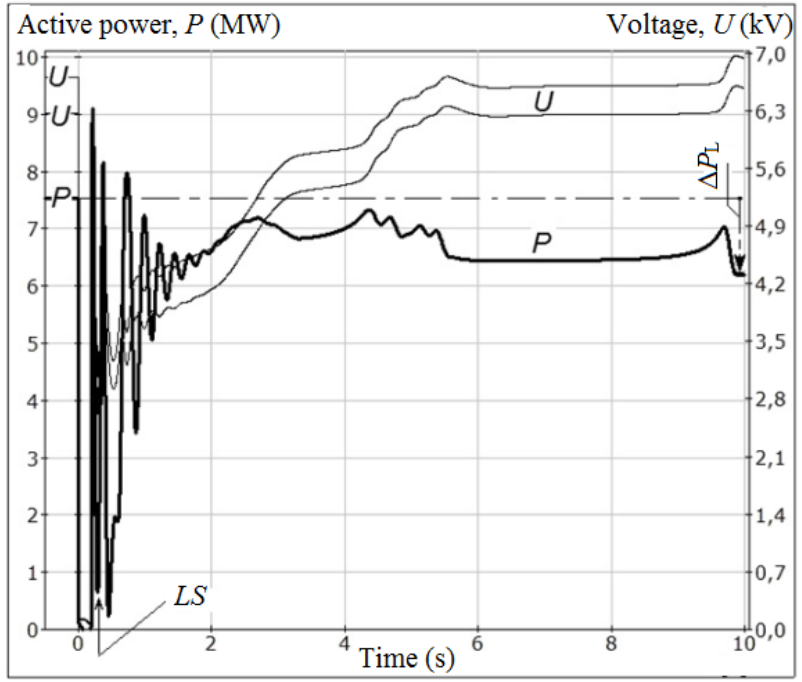

b)

Fig. 3. Comparison of processes that differ in ACVR response speed: a) $\Delta T=4 \mathrm{~s}$; b) $\Delta T=0.3 \mathrm{~s}$.

The delay in unloading from the beginning of the SC $\Delta T$ was set at $4 \mathrm{~s}$ and $0.3 \mathrm{~s}$, as shown in Fig. 3a, 3b, where $U$ is the voltage at two node points of the object, $P$ is the active power coming to the object from the power system, $L S$ is the load shedding, $\Delta P_{\mathrm{n}}$ is the reduction of the post-emergency unloading compared to the pre-emergency one. Volumes of unloading from ACVR in both calculations are set by the limiting ones to prevent the occurrence of a voltage collapse.

The values of the minimum required $\Delta P_{\mathrm{L}}$ shutdowns depend on all load and grid parameters, as well as on the type and location of the SC. The Fig. 4 shows the averaged dependence $\Delta P_{\mathrm{L}}$ of the discharge delay $\Delta T$, for the same object as above.

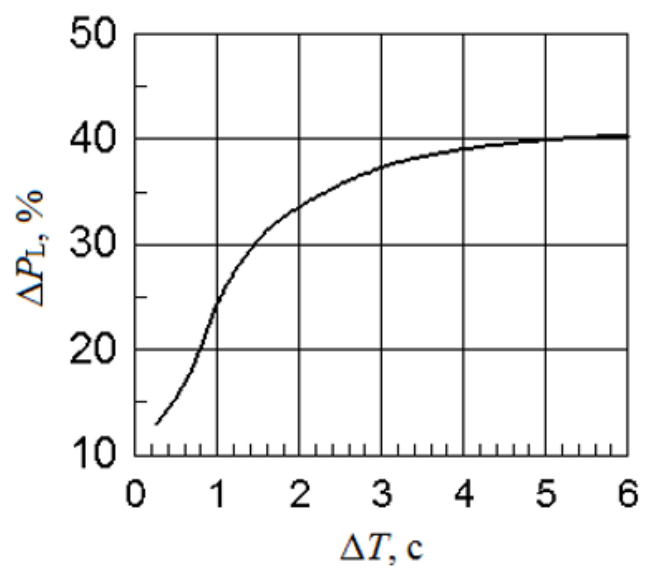

Fig. 4. The effect of the unloading delay on its required value at three-phase short-circuits.

If $\Delta T$ is large, unloading is required, allowing all non-disconnected motors to start from a stationary state. If the value of $\Delta T$ is small, the self-start starts with the slip achieved during the short-circuit, in this case the total self-starting currents are much smaller and the possibility of restoring the normal power supply to the electric receivers is more favorable.

Considering the foregoing, in order to prevent the voltage collapse and not to allow excessive unloading, which causes significant damage to consumers, it is necessary to use signals from start-up and interlock devices of other ECS devices so that the set of all signals indicates the need for high-speed unloading in this circuit-mode situation with sufficient probability. With such a formulation of the problem, it is not possible to consider a high-speed ACVR a local ECS.

\section{Conclusions}

Technological connection of DG objects to distribution electric grids or to internal power supply grids of enterprises inevitably leads to a change in the circuitmode situations in the grid, significantly affecting, in some cases, the power system automatics in operation.

In order to prevent trip failures, as well as unnecessary and false trips of power system automatics, when commissioning the DG objects, it is absolutely necessary to analyze the correctness of the algorithms and the correct selection of the settings of the previously installed automation devices in the adjacent grid.

In addition, it is necessary to check the consistency of the operation algorithms and the settings of the power system automatics in operation with the algorithms of operation and the settings of the new automation devices that must be put into operation during technological connection of the DG object.

In some cases, based on the analysis of circuit-mode situations, it may be necessary to install additional ECS to normalize the parameters of the mode and to keep the most of consumers' receivers in operation, as well as a fundamental change in the algorithms of operation of ECS.

\section{References}

1. Rules for the designing of electrical installations. 7th ed. (Publisjer NTs ENAS, M, 2004).

2. P. Ilyushin, Relay protection and automation, 4, 2836 (2017).

3. GOST R 55105-2012 (Standartinform, M, 2013).

4. Ya. Artsishevskiy, B. Gieev, Energetik, 5, 18-21 (2017).

5. Ya. Artsishevskiy, B. Gieev, University News. «Electromechanics», 6, 37-44 (2017).

6. P. Ilyushin, Energetik, 12, 21-25 (2016).

7. Yu. Gurevich, K. Kabikov, Peculiarities of power supply, aimed at failure-free operation of industrial consumers (ELEKS-KM, M, 2005).

8. GOST 32144-2013 (Standartinform, M, 2014). 